\title{
Development of a nonintermediate-incision ventriculoperitoneal shunt procedure using a nasogastric feeding tube for infant patients with hydrocephalus: technical note
}

\author{
Shuji Hamauchi, MD, Toshitaka Seki, MD, PhD, Toru Sasamori, MD, PhD, and \\ Kiyohiro Houkin, MD, PhD \\ Department of Neurosurgery, Hokkaido University Graduate School of Medicine, Sapporo, Japan
}

\begin{abstract}
Intermediate incisions are considered necessary to pass a catheter tube from the head to the abdomen in ventriculoperitoneal (VP) shunting via a frontal bur hole. However, an intermediate incision can sometimes become dehiscent, resulting in CSF leakage or infection of the shunt system in the early period after shunt implantation, particularly in infant patients. In this article, the authors describe a novel method of VP shunt insertion that does not require an intermediate incision. This nonintermediate-incision VP shunt procedure was performed in 3 infant patients with hydrocephalus and was not associated with any complications. This method can eliminate the intermediate incision, which is a disadvantage of VP shunt insertion via a frontal bur hole.
\end{abstract}

http://thejns.org/doi/abs/10.3171/2015.9.PEDS15464

KEY WORDS ventriculoperitoneal shunt; intermediate incision; pediatrics; hydrocephalus; nasogastric feeding tube; technique

$\mathrm{V}$ ENTRICULOPERITONEAL (VP) shunting is a treatment frequently used in infant patients with hydrocephalus. Children treated with a VP shunt have a higher postoperative shunt infection rate $(9.7 \%)$ than adult patients $(1.7 \%) .{ }^{6}$ Moreover, the VP shunt is the main cause of morbidity and mortality in the treatment of hydrocephalus in infant patients.

VP shunt replacement via the anterior horn of the lateral ventricle usually requires an intermediate incision in the retroauricular region to facilitate curved subcutaneous tunneling from the frontal region of the head to the peritoneal cavity. We have had experiences with cases of shunt infection developing CSF leakage because of a dehiscent wound of the intermediate incision. A previous report indicated that the intermediate incision can be a source of post-shunt complications. ${ }^{5}$ To prevent such complications, we developed a nonintermediate-incision VP shunt procedure.

\section{Methods \\ Patients}

Three infant patients, ranging in age from 1 day to 8 months, underwent insertion of a VP shunt by employing the nonintermediate-incision method. The patients' details are as follows: Case 1 was a 5-month-old girl with myelomeningocele, weighing $7760 \mathrm{~g}$; Case 2 was an 8-monthold girl with holoprosencephaly, weighing $5970 \mathrm{~g}$; and Case 3 was a 1-day-old boy with X-linked hydrocephalus, weighing $2570 \mathrm{~g}$. Case 1 previously underwent removal of the shunt system because of a localized skin infection caused by a dehiscent intermediate-incision wound. Case 
2 suffered repeated shunt infections and underwent shunt removal and external CSF drainage 4 times previously. Case 3 underwent shunt implantation for the first time.

\section{Surgical Technique}

A Codman-Hakim programmable shunt valve, a reusable tubular shunt passer with stylet (inner diameter 3.4 $\mathrm{mm}$, TSQ-400, Tokibo), a flexible surgical probe (length $21 \mathrm{~cm})$, and a nasogastric tube $(8-\mathrm{Fr}, 120 \mathrm{~cm})$ were used for all patients. Patients were placed supine under general anesthesia. Antibiotics were administered intravenously and preoperatively. The surgical site was shaved. Piled sponges were placed under the patient's shoulders to facilitate a straight line from the abdomen to the retroauricular region. The skin was cleaned with a chlorhexidine scrub from the head to the inguinal region and then sterilized with iodine. The entire shunt tract was draped with iodineimpregnated adhesive drape.

A skin incision was made in front of the coronal suture, and a small hole was drilled in the skull. Then, an abdominal skin incision was made and the peritoneal cavity was opened. The shunt passer was passed subcutaneously from the site of the abdominal skin incision to the retroauricular region, and the stylet was withdrawn from the sheath (Fig. 1A and B). Next, the flexible probe was passed from the precoronal incision to the retroauricular site subcutaneously, and the tip of the probe was cannulated into the hole of the passer sheath tip and the probe was removed (Fig. 1C and D). Thus, a subcutaneous tunnel extending over the entire length from the head to the abdomen was opened. Then, a nasogastric tube was inserted into the abdominal opening of the metal sheath. The feeding tube could smoothly pass through the prepared subcutaneous

\section{A}

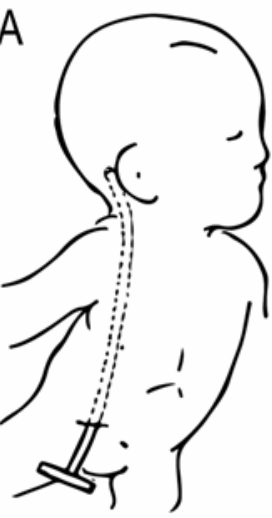

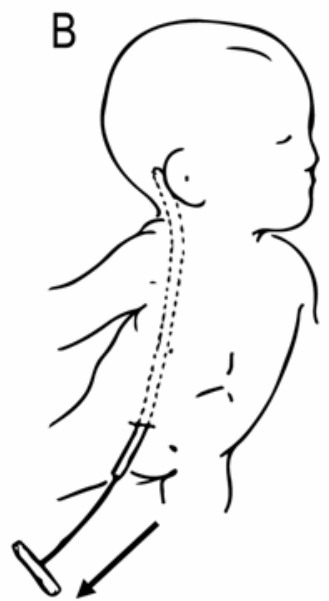

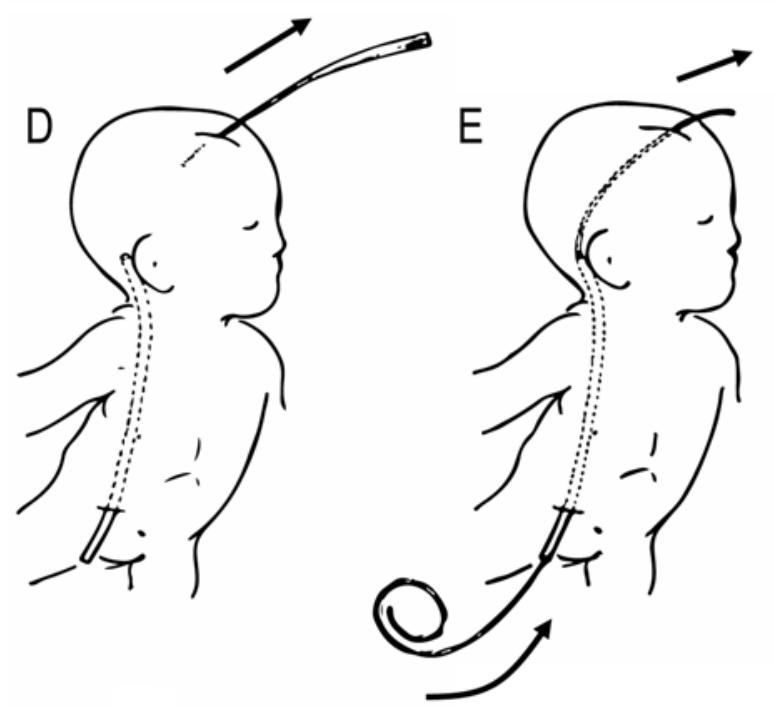

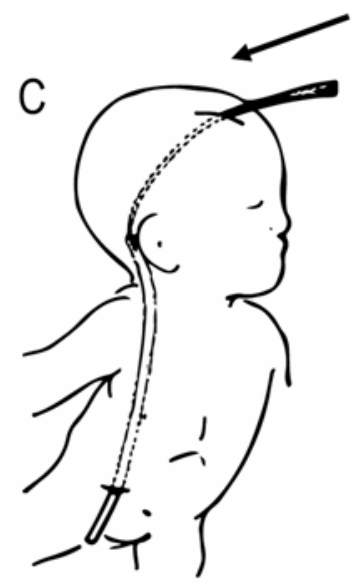

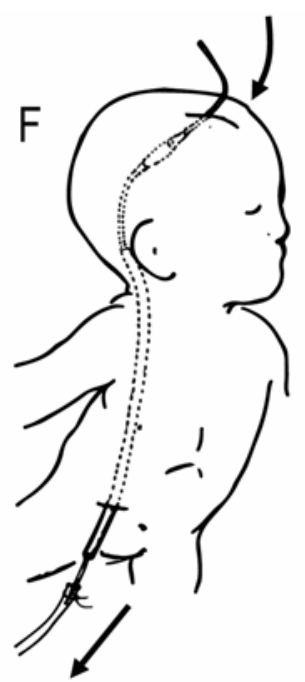

FIG. 1. Illustrations showing the surgical steps involved in this technique. A: Tunneling the shunt passer from the abdomen to the retroauricular site. B: Removal of the stylet. C: Insertion of a flexible surgical probe from the precoronal incision into the opening of the sheath. D: Removal of the surgical probe. E: Passing a nasogastric tube from the abdominal to the precoronal incision. F: Passing the distal end of the shunt system guided with a nasogastric tube. Arrows indicate the direction of movement of the surgical tool. 
tunnel after traversing the retroauricular opening of the metal sheath and reaching the precoronal skin incision (Fig. 1E). The distal end of the shunt system was inserted into the nicked end of the nasogastric tube and secured with sutures (Fig. 2). The distal end of the shunt system was passed through the metal sheath guided by the feeding tube (Fig. $1 \mathrm{~F})$ and the metal sheath was removed. The ventricular catheter was inserted into the lateral ventricle, under ultrasonographic monitoring, from the anterior fontanelle. After confirming CSF outflow from the abdominal end of the shunt system, the abdominal catheter was inserted into the peritoneal cavity, and wound closure was performed in a standard manner.

\section{Results}

The mean follow-up period was 78 days (range 41-104 days) and the mean operative duration was 76 minutes (range 70-81 minutes). No perioperative complications associated with the nonintermediate-incision VP shunt procedure were present. The first patient (Case 1) recovered well without additional medication. The second patient (Case 2) is in a stable condition. However, the third patient (Case 3) experienced subcutaneous CSF leakage along the shunt tube in the cranial area caused by elevated abdominal pressure because of severe constipation. The subcutaneous CSF collection gradually disappeared after treatment of the constipation, and no CSF fistula or shunt infection occurred.

\section{Discussion}

Currently, neurosurgeons typically use a frontal or occipital approach to insert a ventricular catheter in VP shunt surgery. Conflicting reports exist regarding the better approach for reducing the rate of shunt malfunction, ${ }^{1,2}$ and the conclusion is unclear. However, both methods have advantages: the frontal approach has an anatomically consistent landmark, whereas the occipital approach allows for shorter subcutaneous tunneling without an intermediate incision. The selection of the surgical approach will typically depend on the neurosurgeon's experience. At our center, the anterior approach is preferred because it avoids shunt valve placement in the occipital region where the skin tends to be compressed by the weight of the patient's head. Aside from the preference of the frontal approach, we occasionally encountered cases in which the frontal approach was considered more appropriate than the occipital approach because of patient-specific circumstances, e.g., the patient in Case 2 lacked the posterior part of the brain because of a lobar holoprosencephaly.

VP shunt insertion from the frontal horn of the lateral ventricle usually necessitates an intermediate incision in the retroauricular region to assist tunneling of the distal catheter from the frontal region of the head to the abdomen. This intermediate incision is a possible source of complications such as wound dehiscence and CSF fistula. ${ }^{5}$ To avoid making an intermediate incision in the frontal approach, we developed a novel method of VP shunt insertion.

There are a few articles describing a method of VP shunt insertion, avoiding the use of an intermediate inci-

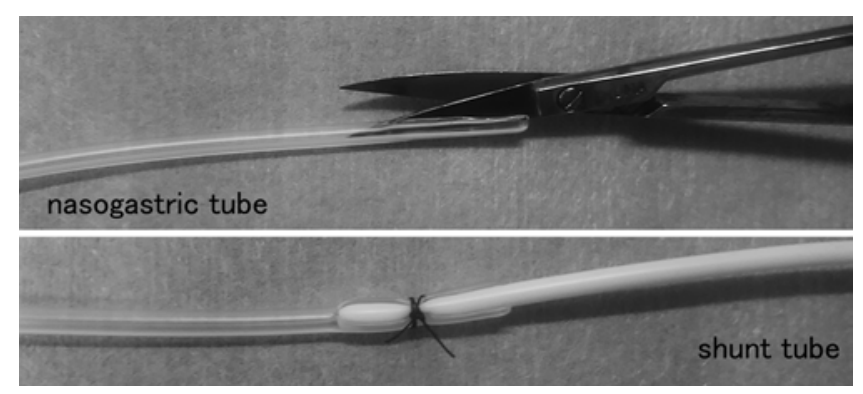

FIG. 2. Upper: The end of a nasogastric tube is nicked with surgical scissors. Lower: The distal end of a shunt tube is inserted into the nicked end of a nasogastric tube and ligated with silk thread.

sion. Kanev and Park reported such a method in $1993,{ }^{3}$ in which they used a malleable shunt passer with a plastic sheath. A large subcutaneous pocket was created from the frontal region toward the superior nuchal line immediately behind the pinna, and a malleable shunt passer was tunneled from the abdominal incision into the subcutaneous pocket of the head; then, a plastic sheath was advanced into the incision of the frontal region though the prepared subcutaneous pocket. These investigators reported an excellent rate of shunt infection of $1.33 \%$ in pediatric patients with hydrocephalus using this method in combination with a "no-touch" technique, minimizing hardware and skin manipulation, and using a double gloving technique. ${ }^{4}$ Moreover, they claimed that operating time was shortened by avoiding an intermediate incision. Sandquist and Selden similarly reported on a "single-pass" tunneling technique using a malleable shunt passer and disposable plastic sheath. ${ }^{5}$ They noted that a single-pass tunneling technique may eliminate one potential site of postoperative complications, such as intermediate-incision CSF leak, dehiscence, or infection. However, these 2 methods cannot be used if a plastic sheath is not used, as we did in our method.

Our method is unique in using a nasogastric tube to pass through the subcutaneous tunnel and guide the distal catheter. The reason we chose a nasogastric tube is its availability in most hospitals, adequate length, and appropriate stiffness for advancing through the subcutaneous tunnel. Moreover, the nasogastric tube has the advantage of lower cost as compared with the disposable plastic sheath. Another advantage of our method is that a large subcutaneous pocket in the head is not required because cannulation of the thin surgical probe from the frontal incision into the opening of the outer sheath is sufficient to introduce a nasogastric tube toward the frontal incision. This can prevent excessive subcutaneous dissection that may facilitate CSF collection.

There are some limitations to our method. Although our method was successfully performed in neonates and infants, its practical use in older children or adults has not been verified. Subcutaneous passing of a nasogastric tube may be difficult in older children or adults because of increased resistance in the subcutaneous space. In addition, our method cannot be achieved without reusable tubular shunt passers whose design allows stylet removal from the proximal end of the sheath. A disposable tubular passer 
is not compatible with our method because it is usually designed to allow stylet removal from the distal end of the sheath. Furthermore, the ultimate success or morbidity rate of our method is unclear because of our small number of cases and relatively short period of observation.

\section{Conclusions}

In this paper we describe a novel method of VP shunt insertion without an intermediate incision, which is one of the disadvantages in the frontal approach. This method is useful for the treatment of neonates or infants with hydrocephalus who plan to undergo a VP shunt insertion via a frontal approach.

\section{References}

1. Albright AL, Haines SJ, Taylor FH: Function of parietal and frontal shunts in childhood hydrocephalus. J Neurosurg 69:883-886, 1988

2. Bierbrauer KS, Storrs BB, McLone DG, Tomita T, Dauser R: A prospective, randomized study of shunt function and infections as a function of shunt placement. Pediatr Neurosurg 16:287-291, 1990-1991

3. Kanev PM, Park TS: The treatment of hydrocephalus. Neurosurg Clin N Am 4:611-619, 1993
4. Kanev PM, Sheehan JM: Reflections on shunt infection. Pediatr Neurosurg 39:285-290, 2003

5. Sandquist MA, Selden NR: A single-pass tunneling technique for CSF shunting procedures. Pediatr Neurosurg 39:254-257, 2003

6. Welch K: Residual shunt infection in a program aimed at its prevention. Z Kinderchir Grenzgeb 28:374-377, 1979

\section{Disclosures}

The authors report no conflict of interest concerning the materials or methods used in this study or the findings specified in this paper.

\section{Author Contributions}

Conception and design: Hamauchi. Acquisition of data: Hamauchi. Analysis and interpretation of data: Hamauchi. Critically revising the article: Seki. Administrative/technical/material support: Sasamori. Study supervision: Houkin.

\section{Correspondence}

Shuji Hamauchi, Department of Neurosurgery, Hokkaido University Graduate School of Medicine, North 15 West 7, Kita, Sapporo 060-8638, Japan. email: hamashuji@yahoo.co.jp. 